\title{
Correlation of Rates of Intramolecular Tunneling Processes, with Application to Some Group V Compounds
}

\author{
R. STEPHEN BERRY \\ Department of Chemistry, The University of Michigan, Ann Arbor, Michigan
}

(Received October 19, 1959)

\begin{abstract}
A method is presented whereby the WKB expression for tunneling frequencies permits correlation of these frequencies for members of homologous series of molecules. The necessary data required are the tunneling rate for one member of the series, and structural parameters and vibrational frequencies for all members of interest. The method is applied to $\mathrm{ND}_{3}, \mathrm{NF}_{3}, \mathrm{PH}_{3}$, and $\mathrm{AsH}_{3}$, using $\mathrm{NH}_{3}$ as the molecule whose frequency is known. The method also is used to support the hypothesis that pseudorotation occurs in the trigonal bipyramids $\mathrm{PF}_{5}$ and $\mathrm{PCl}_{5}$.
\end{abstract}

\section{INTRODUCTION}

$\mathbf{T}$ HE study of intramolecular tunneling motions has focused on the evaluation of two characteristic quantities: the energy level splittings or tunneling frequencies, on one hand, and the heights of energy barriers inhibiting internal motions, on the other. Barrier heights have been examined extensively ${ }^{1}$ because they are sensitive parameters and easily understood whenever the shape of a barrier is well established. And in the class of molecules for which barriers are best known, the barrier shape is well represented by a cosine function. ${ }^{2}$ In contrast, there are many systems which can exhibit intramolecular tunneling for which the barrier shape is not well established, and, in fact, for which tunneling may have been observed for but one or two members of a large series. When one is faced with such a situation, it is more useful to be able to estimate the tunneling frequency than to try to evaluate a barrier height. First, the tunneling frequency or energylevel splitting is often directly observable; second, the barrier height is meaningful only if the form of the potential-energy surface is known. With relatively exact data regarding the energy-level separations, one can try to investigate the shape of the potential in detail. Without such information, the barrier height itself gives very little information about the observable properties and dynamical behavior of a molecule. It therefore becomes useful to be able to estimate tunneling frequencies by some process which does not depend in any critical way on exact shapes or heights of potential barriers.

The ensuing discussion develops a method for estimating tunneling frequencies for members of a homologous series of compounds. The motivation for this treatment is twofold. First, hopefully, it will provide a semiquantitative method whose accuracy is sufficient to allow one to select the appropriate experimental technique for determining any desired tunneling frequency in the laboratory. It should enable one, for example, to choose between infrared, microwave, and

${ }^{1}$ For a review of recent results, see E. B. Wilson, Proc. Natl. Acad. Sci. U. S. 43, 816 (1957).

${ }^{2}$ D. R. Herschbach and J. D. Swalen, J. Chem. Phys. 29, 761 (1958). radio-frequency spectroscopy as possible tools for determining a given tunneling rate. Second, the method is used to develop a possible interpretation of the observed behavior of $\mathrm{PF}_{5}$ and $\mathrm{PCl}_{5}$, an interpretation based on the hypothesis that internal tunneling occurs in these molecules.

In Sec. II, a general treatment is developed in which one obtains tunneling frequencies for any member of a homologous series if the appropriate frequency is known for a single member. The method, based on the Dennison-Uhlenbeck formulation of the WKB approach, ${ }^{3}$ uses as its primary data the structural parameters and vibration frequencies of the various molecules under consideration. Section III is devoted to the application of the method to the pyramidal molecules $\mathrm{ND}_{3}, \mathrm{NF}_{3}, \mathrm{PH}_{3}$, and $\mathrm{AsH}_{3} ; \mathrm{NH}_{3}$ is taken as the molecule whose tunneling frequency is known. This application provides a basis from which one can assess the accuracy of the method and determine which of several possible approximations is most appropriate. Finally, Sec. IV contains a discussion of the possibility of internal motion in $\mathrm{PF}_{5}$ and $\mathrm{PCl}_{5}$, with an attempt to reconcile the available experimental results for these two molecules.

\section{GENERAL FORMULATION}

An expression for the tunneling frequency for an internal motion, like the inversion of ammonia, has been derived by Dennison and Uhlenbeck, ${ }^{3}$ using the WKB method. They find that if the tunneling motion consists predominantly of one normal vibrational mode, and if $\nu=$ tunneling frequency, $\nu_{0}=$ vibrational frequency, $\mu=$ reduced mass for tunneling coordinate, $s=$ displacement along the tunneling coordinate, $s_{0}=s$ at which $E=V(s)$, measured from the barrier maximum, $V(s)=$ potential energy, and $\epsilon=$ total energy, then

where

$$
\nu=\left(\nu_{0} / \pi\right) e^{I},
$$

$$
I=-(1 / \hbar) \int_{-s_{0}}^{s_{0}}\{2 \mu[V(s)-\epsilon]\}^{\frac{1}{1}} d s .
$$

${ }^{3}$ D. M. Dennison and G. E. Uhlenbeck, Phys. Rev. 41, 313 (1932). 


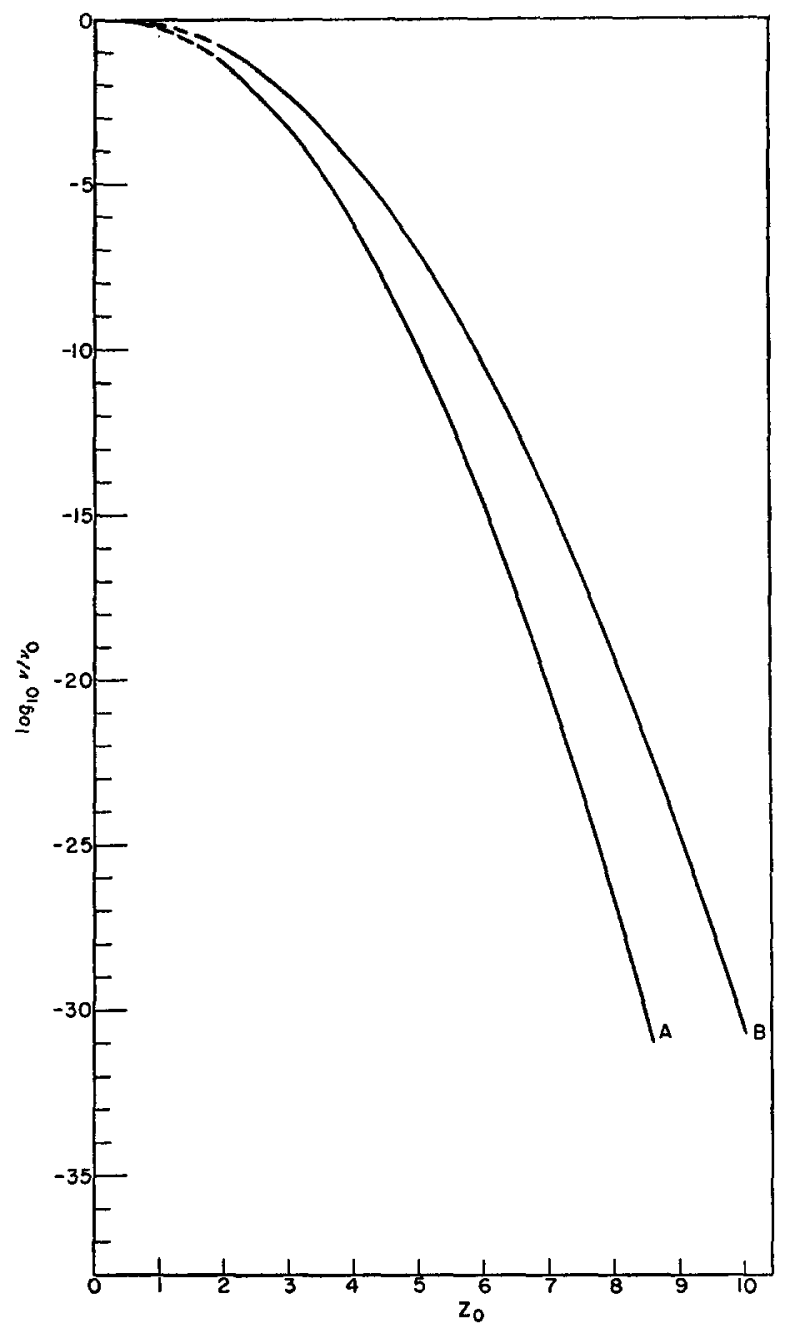

Fig. 1. Logarithmic curves of the ratio of the tunneling frequency $\nu$ to the principal vibrational frequency $\nu_{0}$ along the tunneling coordinate, is the reduced distance $Z_{0}$ (number of classical amplitudes for oscillator of frequency $\nu_{0}$ ) from the potential minimum to the potential barrier maximum. Curve $\mathrm{A}$ represents the quadratic approximation, Curve $B$ the cosine form.

The expression for $I$ can be integrated for a variety of potentials $V$. With the barrier height $V(s=0)$ sufficiently high, so that two or more vibrational levels lie below the barrier, it is convenient for us to change the variable of integration. When $V$ is high, the lowest vibrational state is approximately that of a harmonic oscillator with frequency $\omega_{0}=2 \pi \nu_{0}$; we introduce the variable

$$
Z=\left(\mu \omega_{0} / \hbar\right)^{\frac{1}{2}} r
$$

where $r$ is the displacement along the tunneling coordinate with the origin at a potential minimum. Then if $R$ is the distance from the origin to the potential maximum, we let

$$
Z_{0}=\left(\mu \omega_{0} / \hbar\right)^{\frac{1}{2}} R
$$

The dimensionless variable $Z$ has as its unit the classical amplitude of oscillation for an oscillator of frequency $\omega_{0}$, in its lowest quantum state. Therefore at $s_{0}, Z=1$. The expression for $I$ becomes

$$
I=-2\left(2 / \hbar \omega_{0}\right)^{\frac{1}{2}} \int_{1}^{Z_{0}}[V(Z)-\epsilon]^{\frac{1}{d}} d Z
$$

If we let $\epsilon=\frac{1}{2} \hbar \omega_{0}$, then

$$
I=-2 \int_{1}^{z_{0}}[V(Z) / \epsilon-1]^{\frac{1}{2}} d Z,
$$

which is a particularly useful form to use for the integration. For most reasonable potentials, $V$ has a quadratic leading term, so that the tunneling frequency will vary roughly as

$$
\nu \sim \exp -Z_{0}^{2} f\left(Z_{0}\right),
$$

where $f\left(Z_{0}\right)$ is constant or slowly varying compared with $Z_{0}^{2}$. If $V$ is formed from two intersecting parabolas, then the integrated expression gives, for the $n$th vibrational level,

$$
\begin{aligned}
\mathrm{I}_{q}=-\left[Z_{0}\left(Z_{0}^{2}-2 n-1\right)^{\frac{1}{2}}-(2 n+1)\right. & \log Z_{0} \\
& \left.+\left(Z_{0}^{2}-2 n-1\right)^{\frac{1}{2}}\right] .
\end{aligned}
$$

Alternatively, if $V$ iș represented as a cosine function,

$$
V_{\text {cos }}=\frac{1}{2} V_{0}\left[1-\cos \left(\pi Z / Z_{0}\right)\right] \text {, }
$$

then for a state of energy $\epsilon$,

$$
I_{\mathrm{oos}}=\left(16 \sqrt{2} / \pi^{3}\right) Z_{0}^{2}\left[E(k)-\left(\epsilon / V_{0}\right) K(k)\right],
$$

where $k=\left[\left(V_{0}-\epsilon\right) / V_{0}\right]^{\frac{1}{2}}$ and $E(k)$ and $K(k)$ are the complete elliptic integrals of the second and first kinds, respectively. Approximating $\epsilon$ as $\hbar \omega_{0} / 2$ as we have done, with $\omega_{0}$ the circular frequency for a harmonic oscillator

TABLE I. Data used for treatment of $\mathrm{NH}_{3}$-like molecules.

\begin{tabular}{lcccc}
\hline \hline Molecule & $r_{\mathrm{X}-\mathrm{Y}}(\mathrm{A})$ & $\theta^{\mathrm{a}}$ & $\nu_{2}^{\mathrm{b}}\left(\mathrm{cm}^{-1}\right)$ & $\mu(\mathrm{amu})$ \\
\hline $\mathrm{NH}_{3}$ & $1.014^{\mathrm{c}}$ & $25^{\circ} 2^{\prime \mathrm{c}}$ & $1055^{\mathrm{c}, \mathrm{d}}$ & $2.47^{\circ}$ \\
$\mathrm{ND}_{3}$ & $1.014^{\mathrm{o}}$ & $25^{\circ} 2^{\prime \mathrm{c}}$ & $805^{\mathrm{d}}$ & $4.20^{\circ}$ \\
$\mathrm{NF}_{3}$ & $1.371^{\circ}$ & $26^{\circ} 3^{\prime \mathrm{o}}$ & $505^{\mathrm{f}}$ & 11.24 \\
$\mathrm{PH}_{3}$ & $1.42^{\mathrm{g}}$ & $32^{\circ} 45^{\prime \mathrm{c}}$ & $992^{\mathrm{b}}$ & 2.73 \\
$\mathrm{AsH}_{3}$ & $1.52^{\mathrm{s}}$ & $33^{\circ} 50^{\prime \mathrm{s}}$ & $974^{\mathrm{i}}$ & 2.88 \\
\hline
\end{tabular}

$\mathrm{a} \theta$ is the angle between the $\mathrm{X}-\mathrm{Y}$ bond and the plane perpendicular to the molecular symmetry axis.

b Frequencies are observed values except for $\mathrm{NH}_{3}, \mathrm{ND}_{3}$, and $\mathrm{AsH}_{3}$, where harmonic frequencies were used (see reference 11 and $d$, below).

- See reference 12.

d D. M. Dennison, Revs. Modern Phys. 12, 175 (1940).

C P. Kisliuk, J. Chem. Phys. 22, 86 (1954).

f Bailey, Hale, and Thompson, J. Chem. Phys. 5, 274 (1937).

c C. C. Loomis and M. W. P. Strandberg, Phys. Rev, 81, 798 (1951); L. S. Bartell and R. C. Hirst, J. Chem. Phys. 31, 449 (1959).

h V. M. McConaghie and H. H. Nielsen, J. Chem. Phys. 21, 1836 (1953).

i V. M. McConaghie and H. H. Nielsen, Phys. Rev. 75, 633 (1949). 
TABLE II. Parameters, tunneling frequencies and times for $\mathrm{NH}_{3}$-like molecules. Upper values are taken with $\mathrm{Y}_{3}$ moving as a rigid triangle, lower values with the $\mathrm{X}-\mathrm{Y}$ bond length held constant.

\begin{tabular}{|c|c|c|c|c|c|c|}
\hline \multirow[b]{2}{*}{ Molecule } & \multicolumn{3}{|c|}{ Quadratic approximation } & \multicolumn{3}{|c|}{ Cosine approximation } \\
\hline & $Z_{0}$ & $\nu\left(\sec ^{-1}\right)$ & $\tau(\mathrm{sec})$ & $Z_{0}$ & $\nu\left(\sec ^{-1}\right)$ & $\tau(\mathrm{sec})$ \\
\hline $\mathrm{NH}_{3}$ & 2.88 & & & 3.42 & $\begin{array}{c}2.3786 \times 10^{10} \\
(\text { obs.) })^{3}\end{array}$ & \\
\hline $\mathrm{ND}_{3}$ & 3.29 & $1.84 \times 10^{9}$ & $2.7 \times 10^{-10}$ & 3.91 & $\begin{array}{l}1.43 \times 10^{9} \\
1.6 \times 10^{9} \\
(\text { obs.) } \\
1.25 \times 10^{9} \\
(\text { calc.) }\end{array}$ & $3.5 \times 10^{-10}$ \\
\hline $\mathrm{NF}_{3}$ & $\begin{array}{l}5.96 \\
5.98\end{array}$ & $\begin{array}{l}0.031 \\
0.023\end{array}$ & $\begin{array}{l}16 \\
22\end{array}$ & $\begin{array}{l}7.08 \\
7.10\end{array}$ & $\begin{array}{l}0.015 \\
0.012\end{array}$ & $\begin{array}{l}33 \\
42\end{array}$ \\
\hline $\mathrm{PH}_{3}$ & $\begin{array}{l}5.26 \\
5.38\end{array}$ & $\begin{array}{c}156 . \\
45.2\end{array}$ & $\begin{array}{l}3.2 \times 10^{-3} \\
1.1 \times 10^{-2}\end{array}$ & $\begin{array}{l}6.25 \\
6.39\end{array}$ & $\begin{array}{l}78.5 \\
21.4\end{array}$ & $\begin{array}{l}6.4 \times 10^{-3} \\
2.3 \times 10^{-2}\end{array}$ \\
\hline $\mathrm{AsH}_{3}$ & $\begin{array}{l}5.90 \\
6.06\end{array}$ & $\begin{array}{l}0.130 \\
0.020\end{array}$ & 25.9 & $\begin{array}{l}7.00 \\
7.19\end{array}$ & $\begin{array}{l}5.9 \times 10^{-2} \\
1.5 \times 10^{-3}\end{array}$ & 53 \\
\hline
\end{tabular}

See reference 4.

b See reference 4 or 8 .

whose force constant is the same as that of $V_{\text {cos }}$, then Eq. (9) can be simplified by the substitution

$$
\epsilon / V_{0}=\pi^{2} / 4 Z_{0}^{2} \text {. }
$$

In both these cases, the tunneling frequency is indeed roughly dependent on $\exp \left(-A Z_{0}^{2}\right)$. This suggests that one might correlate tunneling frequencies for similar molecules by assuming any reasonable potential, providing (a) one knows the tunneling frequency for one molecule of the set, and (b) the tunneling coordinate is dominated by one normal mode, or at least by modes of comparable frequency. Then the quantity $Z_{0}$ becomes a parameter of correlation only, and depends on the potential form chosen; at the same time the form of $Z_{0}$ demands that for any two substances $\mathrm{A}$ and $\mathrm{B}$, their $Z_{0}$ parameters be related by the condition that

$$
Z_{0}(\mathrm{~A}) / Z_{0}(\mathrm{~B})=\left(\mu_{\mathrm{A}} \omega_{0 \mathrm{~A}} / \mu_{\mathrm{B}} \omega_{0 \mathrm{~B}}\right)^{\frac{1}{2}}\left(R_{\mathrm{A}} / R_{\mathrm{B}}\right) .
$$

The masses $\mu_{\mathrm{A}}$ and $\mu_{\mathrm{B}}$, the frequencies $\omega_{0 \mathrm{~A}}$ and $\omega_{0 \mathrm{~B}}$, and the distances $R_{\mathrm{A}}$ and $R_{\mathrm{B}}$ are determined in a great number of cases by the symmetry, the structural parameters, and the normal vibration frequencies. Consequently one can pick a likely analytical form for $V$, and reading values from a plot of $\log _{10}\left(\nu / \nu_{0}\right)$ is $Z_{0}$, determine the approximate tunneling rate for any member of a homologous series, knowing previously the rate for one member only.

In Fig. 1 are plotted the curves of $\log _{10}\left(\nu / \nu_{0}\right)$ vs $Z_{0}$ for the cosine and parabolic potentials. The two curves are nearly parallel, and the similarity of results they give is demonstrated in Sec. III.

\section{APPLICATION TO SOME XY $\mathrm{XY}_{3}$ PYRAMIDAL MOLECULES}

The tunneling process corresponding to the inversion of $\mathrm{NH}_{3}$ and similar molecules provides a straight- forward test of the method developed in Sec. II. Inversion splittings are well known for $\mathrm{NH}_{3}$ and $\mathrm{ND}_{3}$, but have not been observed spectroscopically for any heavier homologs. ${ }^{4}$ The problem of inversion frequencies has been treated by several authors. ${ }^{5-11}$ Of principal interest have been the barrier shape and height although there has been one method developed to correlate the $\mathrm{NH}_{3}, \mathrm{PH}_{3}$, and $\mathrm{AsH}_{3}$ barriers and tunneling frequencies. ${ }^{11}$ The most accurate potential for which both $\mathrm{NH}_{3}$ and $\mathrm{ND}_{3}$ tunneling frequencies are known is the one developed by Manning; ${ }^{8}$ this, together with the observed results, will serve as a standard for our approximation method.

The tunneling frequencies of $\mathrm{ND}_{3}, \mathrm{NF}_{3}, \mathrm{PH}_{3}$, and $\mathrm{AsH}_{3}$ were calculated four ways; the two potentials were each tried with the assumptions that in $\mathrm{XY}_{3}$, the $\mathrm{XY}$ bond lengths remain constant, or alternatively, that $\mathrm{Y}_{3}$ moves as a rigid triangle. The tunneling coordinate of $\mathrm{NH}_{3}$ almost certainly is dominated by one normal coordinate, the $\nu_{2}$ vibration. This vibration in turn, expressed in valence force-field coordinates, consists principally of the $S_{2}$ angle-bending vibration ${ }^{12}$ :

$$
S_{1} / S_{2}=1 / 10.96 \text {, }
$$

based on the data contained in Table $\mathrm{I}$. (This value is

${ }^{4}$ C. H. Townes and A. L. Schawlow, Microwave Speciroscopy (McGraw-Hill Book Company, Inc., New York, 1955).

${ }^{5} \mathrm{~F}$. Hund, Z. Physik 43, 805 (1927).

${ }^{6}$ P. M. Morse and E. C. G. Stueckelberg, Helv. Phys. Acta 4, 337 (1931).

${ }^{7}$ N. Rosen and P. M. Morse, Phys. Rev. 42, 210 (1932).

8 M. F. Manning, J. Chem. Phys. 3, 136 (1935).

${ }^{9}$ F. T. Wall and G. Glockler, J. Chem. Phys. 5, 314 (1937).

${ }_{10}$ R. R. Newton and L. H. Thomas, J. Chem. Phys, 16, 310 (1948).

${ }^{11}$ C. C. Costain and G. B. B. M. Sutherland, J. Phys. Chem. 56, 321 (1952).

${ }_{12} \mathrm{G}$. Herzberg, Infrared and Raman Spectra (D. Van Nostrand Company, Inc., Princeton, New Jersey, 1945). See especially, pp. 154-157, 175-177. 

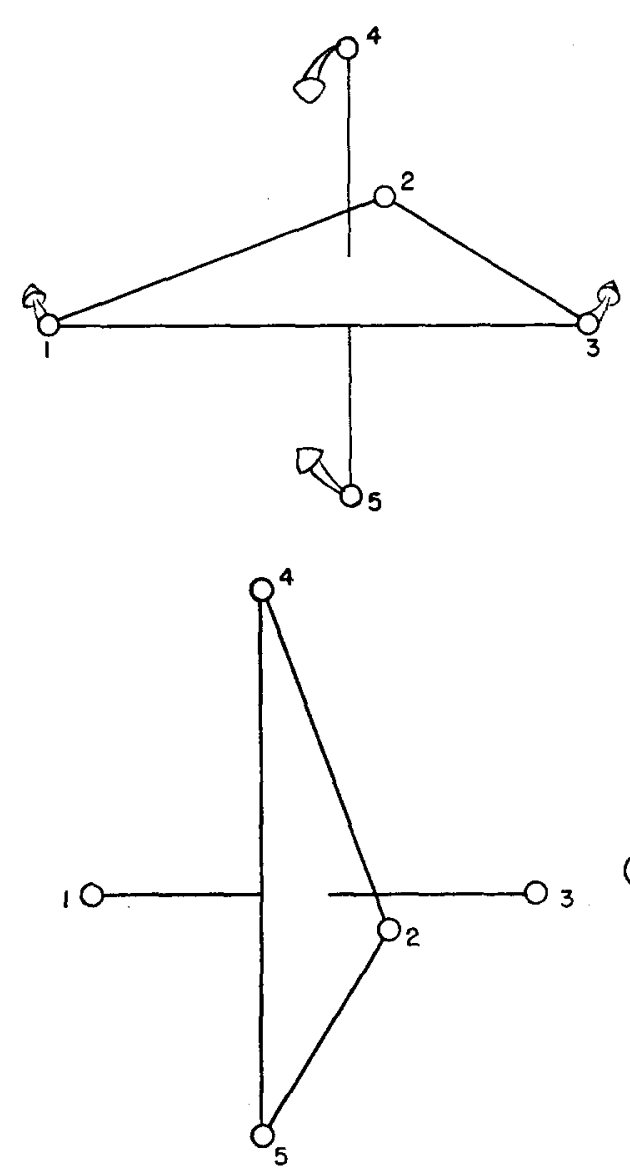

(b)

FIG. 2. Schematic representation of pseudorotation in the trigonal bipyramid.

not in exact agreement with that of reference 11 ; by using this value for $S_{1} / S_{2}$, the potential computed in the Costain-Sutherland treatment is about $100 \mathrm{~cm}^{-1}$ less than the value reported.) We therefore take the $\nu_{2}$ vibration as the one to be substituted for $\nu_{0}$ in Eq. (1) and (11); as Costain and Sutherland ${ }^{11}$ point out, it is more appropriate to use the normal vibration frequency of the state in question than the observed infrared frequency. This has been done whenever normal frequencies were available. The effective masses have been taken as constants, with

$$
\mu=3 m_{\mathbf{x}} m_{\mathbf{y}} /\left(m_{\mathbf{x}}+3 m_{y}\right) \text {. }
$$

The expression is almost unaffected by the angledependent contribution which would appear in a rigorous treatment. Structural parameters, effective masses, and vibration frequencies are given in Table I.

Table II gives the results obtained with the four sets of assumptions. Several points should be noted. First, the frequencies found for $\mathrm{ND}_{3}$, with quadratic and cosine potentials of 1840 and $1430 \mathrm{mc}$, respectively, should be compared with the observed value of 1600 $\mathrm{mc}$ and with the value computed by Manning as 1250 mc. Second, the tunneling frequencies for $\mathrm{PH}_{3}$ and $\mathrm{AsH}_{3}$ are much closer to each other than the frequency of $\mathrm{NH}_{3}$ to either. This seems quite reasonable in light of the similarities in bond lengths, angles, effective masses, and frequencies. It also is interesting that $\mathrm{NF}_{3}$, with its rather flat structure, tunnels at a rate very close to $\mathrm{AsH}_{3}$; in the former, the effective mass is the principal cause of the low frequency; in the latter, the actual distance which the hydrogens must move is the major source of the slow rate. Finally, the most important of all, we see that the various approximations all lead to the same order of magnitude in their predictions, so that one might have enough confidence in the method to estimate rates for the purpose of designing experiments.

\section{PENTAHALIDES OF PHOSPHORUS}

The molecules $\mathrm{PF}_{5}$ and $\mathrm{PCl}_{5}$ have been the subject of a variety of studies which have suggested the possibility that they might exhibit internal motions. Both molecules are trigonal bipyramids so that, at any instant, the halogen atoms occupy two kinds of sites; three are assigned to equatorial positions, and the other two to polar positions. The $\mathrm{F}^{19}$-resonance spectrum of $\mathrm{PF}_{5}$ has been observed by Gutowsky, McCall, and Slichter. ${ }^{13}$ They report only a single fluorine resonance peak (split into a doublet due to $\mathrm{P}-\mathrm{F}$ coupling), so that either the five fluorines are equivalent to a measurement as slow as NMR, or the chemical shifts and $\mathrm{P}-\mathrm{F}$ couplings are accidentally identical for fluorines at both kinds of sites. The latter possibility is rather unlikely, particularly in light of the data to be cited regarding $\mathrm{PCl}_{5}$, so it will not be considered further.

The infrared spectrum of $\mathrm{PF}_{5}$ has been observed by Gutowsky and Liehr, ${ }^{14}$ who find an apparently normal spectrum for a trigonal bipyramid. This suggests that the fluorine atoms may exchange at a rate slow compared with the time necessary to establish sharp vibrational levels, and fast compared with the inverse line widths associated with nuclear resonance measurements. Hence any exchange process which the fluorines

TABLE III. Data used for treatment of $\mathrm{PF}_{5}$ and $\mathrm{PCl}_{5}$.

\begin{tabular}{|c|c|c|c|}
\hline Molecule & $r_{\mathrm{X}-\mathrm{Y}}$ of $\mathrm{XY}_{2}(\mathrm{~A})$ & $r_{\mathrm{XY}}$ of $\mathrm{XY}_{3}(\mathrm{~A})$ & $\nu_{0}\left(\mathrm{~cm}^{-1}\right)$ \\
\hline $\mathrm{PF}_{3}$ & $1.57 \mathrm{~s}$ & $1.57^{\mathrm{a}}$ & $\begin{array}{c}534^{\circ} \\
\text { or } \\
\sim 180 \text { (assumed) }\end{array}$ \\
\hline $\mathrm{PCl}_{5}$ & $2.04^{\mathrm{b}}$ & $2.18^{\mathrm{b}}$ & $\begin{array}{l}335^{\mathrm{d}} \\
\text { or }^{\mathrm{d}} \\
100^{\mathrm{d}}\end{array}$ \\
\hline
\end{tabular}

a L. O. Brockway and J. Y. Beach, J. Am. Chem. Soc. 60, 1836 (1938).

b See reference 16.

- See reference 14.

d See reference 15

${ }^{13}$ Gutowsky, McCall, and Slichter, J. Chem. Phys. 21, 279 (1953).

14 H. S. Gutowsky and A. D. Liehr, J. Chem. Phys. 20, 1652 (1953). 
TABLE IV. Parameters, tunneling frequencies and times for $\mathrm{PF}_{5}$ and $\mathrm{PCl}_{5}$. Upper values are derived using the high-frequency values of Table III, lower values using the lower frequencies.

\begin{tabular}{cccccccc}
\hline & \multicolumn{3}{c}{ Quadratic approximation } & \multicolumn{4}{c}{ Cosine approximation } \\
Molecule & $Z_{0}$ & $\nu\left(\sec ^{-1}\right)$ & $\tau$ & $Z_{0}$ & $\nu\left(\sec ^{-1}\right)$ & $\tau$ \\
\hline $\mathrm{PF}_{5}$ & 4.37 & $10^{5}$ (assumed) & & 5.17 & $10^{5}$ (assumed) \\
$\mathrm{PCl}_{5}$ & 6.41 & $2.4 \times 10^{-5}$ & $5.8 \mathrm{hr}$ & 7.59 & $9.6 \times 10^{-6}$ & $12.8 \mathrm{hr}$ \\
& & $1.2 \times 10^{-4}$ & $41 \mathrm{sec}$ & & $6 \times 10^{-4}$ & $1.4 \mathrm{~min}$ \\
\hline
\end{tabular}

undergo should occur at a frequency between about $10^{-8}$ and $10^{-2}$ sec.

Now we turn to the data regarding $\mathrm{PCl}_{5}$. Infrared and Raman spectra have been taken and analyzed by Wilmshurst and Bernstein. ${ }^{15}$ The structure is known from electron diffraction work of Rouault. ${ }^{16}$ The possibility of internal exchange is suggested in the isotopic exchange studies of Downs and Johnson. ${ }^{77,18}$ They report that three chlorines are capable of very rapid exchange, giving complete equilibration in $5 \mathrm{sec}$ or less; the other two chlorine nuclei do exchange, but attain $90 \%$ of their equilibrium isotopic concentration only after $100 \mathrm{~min}$. Presumably the two slowly exchanging chlorines are those occupying the polar positions, and the rate difference truly is due to a difference in reactivity of the two kinds of sites. By inference, then, it is likely that the two kinds of sites in $\mathrm{PF}_{5}$ are also different chemically, so that instantaneous determination of electron density around the two positions would yield two different results. The reactivity of chlorines in $\mathrm{PCl}_{5}$ thus lends credence to the hypothesis that some kind of exchange process establishes the $\mathrm{F}^{19}$ equivalence in $\mathrm{PF}_{5}$ for NMR observations.

One particularly attractive possible mechanism for the exchange process in $\mathrm{PF}_{5}$ and $\mathrm{PCl}_{5}$ is a purely internal pseudorotation as indicated in Fig. 2. The motion leaves the molecule in a rotated and permuted form of its original state. ${ }^{19}$ If the internal exchange or pseudorotation hypotheis is tenable, the disparity between the necessary $\mathrm{PF}_{5}$ and $\mathrm{PCl}_{5}$ exchange rates must be rationalized. For this we may use the method developed in Sec. II.

The data, including the vibration frequencies of possible importance, are collected in Table III. It would be desirable to be able to attribute the tunneling coordinate with a single normal mode, but unfortunately this cannot be done necessarily. In addition to the problem of finding normal coordinates for $\mathrm{PF}_{5}$, whose spectrum is not entirely known, there seems to be some doubt about the assignments of the $\nu_{6}$ and $\nu_{7} e^{\prime}$ vibra-

\footnotetext{
${ }^{15} \mathrm{~J}$. K. Wilmshurst and H. J. Bernstein, J. Chem. Phys. 27, $661(1957)$.

${ }_{16}$ M. Rouault, Ann. phys. 14, 78 (1940).

${ }_{17} \mathrm{~J}$. J. Downs and R. E. Johnson, J. Chem. Phys. 22, 143 (19.54).

${ }^{18} \mathrm{~J}$. J. Downs and R. E. Johnson, J. Am. Chem. Soc. 77, 2098 (1955).

${ }_{19}$ The author is indebted to Dr. F. T. Smith who first pointed out to him the existence of the pseudorotation operation of a trigonal bipyramid.
}

tions. The tentative assignment of Gutowsky and Liehr ${ }^{14}$ suggested that the $\mathrm{PX}_{3}$ deformation is $\nu_{6}$, at $534 \mathrm{~cm}^{-1}$ for $\mathrm{PF}_{5}$. The normal coordinate analysis of Wilmshurst and Bernstein ${ }^{15}$ gives $\nu_{6}$ as the $\mathrm{PX}_{2}$ polar bend in $\mathrm{PCl}_{5}$ at $335 \mathrm{~cm}^{-1}$, and $\nu_{7}$ as the $\mathrm{PX}_{3}$ deformation at $100 \mathrm{~cm}^{-1}$. The assignments of Wilmshurst and Bernstein however, may be questioned because they found it necessary to call the $394-\mathrm{cm}^{-1}$ band degenerate, assigning it to two vibrations of the same symmetry, the $\nu_{1}$ and $\nu_{2} a_{1}^{\prime}$ modes. It is conceivable but surprising that such a combination shows no significant splitting. In any case, we can compute the data of interest using both the 534 and $335-\mathrm{cm}^{-1}$ bands and the assumed frequency of $180 \mathrm{~cm}^{-1}$ for $\mathrm{PF}_{5}$ with the observed $100-\mathrm{cm}^{-1} \mathrm{PCl}_{5}$ band. These two combinations give results which are all relatively satisfactory for reconciling the tunneling rates.

We assume that the tunneling frequency of $\mathrm{PF}_{5}$ is halfway between the limits set by its normal infrared spectrum and its single NMR peak; namely, we assume that $\nu\left(\mathrm{PF}_{5}\right) \sim 10^{5} \mathrm{sec}^{-1}$. Then with the assistance of Fig. 1 , we compute the corresponding $Z_{0}$. To calculate the $\mathrm{PCl}_{5}$ tunneling frequency, we need to know the distance traveled by the point representing the tunneling particle. We supposed that the halide moves along a straight line from one kind of position to the other, so that the $\mathrm{P}-\mathrm{X}$ distance varies linearly with displacement. Then the average $\mathrm{P}-\mathrm{X}$ distance along this coordinate is used to calculate $Z_{0}$ for $\mathrm{PCl}_{5}$. Note that in contrast to the $\mathrm{NH}_{3}$-like molecules, the moving nuclei in trigonal bipyramids all must travel through the same angle.

The results of the calculations are shown in Table IV. It is rather surprising and somewhat fortuitous that the various approximations do give tunneling times which bracket the characteristic time which would obtain if pseudorotation governed the slow exchange rate in $\mathrm{PCl}_{5}$. The important conclusion to be drawn is simply that the hypothesis of pseudorotation in $\mathrm{PF}_{5}$ and $\mathrm{PCl}_{5}$ is consistent with the experimental data now available. Further experimentation is clearly necessary to confirm or reject the hypothesis.

Other related systems such as $\mathrm{SF}_{4}$ and $\mathrm{ClF}_{3}$ have been studied by Muetterties and Phillips. ${ }^{20,21}$ It has been

${ }^{20}$ E. L. Muetterties and W. D. Phillips, J. Am. Chem. Soc. 79, $322(1957)$.

${ }^{21}$ E. L. Muetterties and W. D. Phillips, J. Am. Chem. Soc. 81, 1084 (1959). 
pointed out that these compounds are capable of exhibiting pseudorotation, ${ }^{22}$ and the results of NMR studies by Muetterties and Phillips ${ }^{20,21}$ are consistent with this hypothesis. Chemical exchange is, however, at least as likely for these molecules. ${ }^{23}$ Therefore we do not suggest that pseudorotation is necessarily the explanation for the observed line narrowings which appear as sample temperatures are increased. It is important, nonetheless, to note that the results of Muetterties and Phillips require that the pseudorotation times for $\mathrm{SF}_{4}$ and $\mathrm{ClF}_{3}$ be considerably longer than for $\mathrm{PF}_{5}$. This is rationalized easily by the following argument. In $\mathrm{PF}_{5}$, the phosphorus atom lies at the center of gravity and, therefore, need not move during the course of pseudorotation. Since $\mathrm{SF}_{4}{ }_{4}^{24,25}$ and $\mathrm{ClF}_{3}^{26,27}$ are not symmetrical structures, the heavy atoms do not lie at the molecular centers of gravity and therefore must participate in pseudorotation. The large effective masses for motion along the tunneling coordinates would depress the tunneling rates, presumably by several orders of magnitude.

\footnotetext{
${ }^{22}$ R. S. Berry, Revs. Modern Phys. (to be published).

${ }^{23}$ M. T. Rogers and J. J. Katz, J. Am. Chem. Soc. 74, 1375 (1952).

${ }^{24}$ Dodd, Woodward, and Roberts, Trans. Faraday Soc. 52, 1052 (1955).

${ }^{25}$ Cotton, George, and Waugh, J. Chem. Phys. 28, 994 (1958).

${ }^{26}$ R. D. Burbank and F. N. Bensey, J. Chem. Phys. 21, 602 (1953).

${ }_{27}$ D. F. Smith, J. Chem. Phys. 21, 609 (1953).
}

\section{SUMMARY}

A method has been presented whereby the tunneling frequencies $\nu$ for members of a homologous series of molecules may be estimated. The method depends on knowledge of the tunneling rate for one member of the series, and the vibrational frequencies and structure for this molecule and for any molecule whose tunneling frequency is desired. The method, useful only for finding orders of magnitude of $\nu$, depends on the insensitivity of the tunneling frequency to barrier shape, and on the assumption that barrier shapes for the various members of the series are similar.

The method is applied to the $\mathrm{NH}_{3}$-like molecules $\mathrm{ND}_{3}, \mathrm{NF}_{3}, \mathrm{PH}_{3}$, and $\mathrm{AsH}_{3}$. The $\mathrm{ND}_{3}$ rate is close to the observed value if either a quadratic or cosine potential is assumed. The $\mathrm{PH}_{3}$ rate is calculated to be of the order of $10 \mathrm{sec}^{-1}$, while the $\mathrm{NF}_{3}$ and $\mathrm{AsH}_{3}$ rates are of the order of $1 \mathrm{~min}^{-1}$.

The method also is applied to correlate the rates of the hypothesized pseudorotation of $\mathrm{PF}_{5}$ and $\mathrm{PCl}_{5}$. If a characteristic time of $10^{-5} \mathrm{sec}$ is assumed for $\mathrm{PF}_{5}$, then the computed time for $\mathrm{PCl}_{\mathfrak{5}}$ lies between $\frac{1}{2}$ min and 12 hrs. The hypothesis of pseudorotation therefore is consistent with the spectra and exchange experiments which have been performed on these two molecules.

\section{ACKNOWLEDGMENT}

The author would like to thank Professor R. B. Bernstein and Professor K. T. Hecht for their helpful comments. 